\title{
De la definición semántica a los ítems a través de las tablas de especificaciones: elabora- ción de la Escala sobre Representación de la Enfermedad, ERCE.
}

\author{
Macarena de los Santos-Roig* y Cristino Pérez-Meléndez
}

Departamento de Metodologia de las Ciencias del Comportamiento. Facultad de Psicologia. Universidad de Granada

\begin{abstract}
Resumen: En este trabajo se presenta la definición semántica del constructo Representación de la Enfermedad, así como las Tablas de Especificaciones del Test y de los Items a partir de las que se desarrolló la prueba ERCE, sobre Representación Cognitiva de la Enfermedad. La ERCE está compuesta por una escala de identidad, otra de causas, consecuencias, control y curso, y todas ellas evalúan creencias relativas a la enfermedad. La idea central de este trabajo es hacer hincapié en la importancia de estos aspectos antes de la construcción de los ítems y mostrar un ejemplo de ello, ya que muchas veces son pocos los trabajos en este sentido. Se presentan también los ítems desarrollados y algunos resultados sobre el análisis de ítems cualitativo de las escalas de la ERCE, así como las modificaciones sobre los ítems iniciales. Finalmente, se presenta la escala ERCE completa.

Palabras clave: Representación de la Enfermedad; definición semántica; tablas de especificaciones; construcción de ítems; juicio de expertos; validez de contenido; análisis de ítems; escala ERCE.
\end{abstract}

\section{Introducción: Justificación del trabajo}

En numerosas ocasiones es patente la dificultad para encontrar trabajos sobre la elaboración de un instrumento para la medida psicológica donde se muestren ejemplificadas las fases que han de completarse, necesariamente, desde el establecimiento de la definición semántica hasta la construcción de los ítems.

En los trabajos que versan sobre la elaboración de instrumentos de medición psicológica, es común encontrar los ítems ya desarrollados (muchas veces imitando lo que aparece en la versiones anglosajonas de las mismas escalas) y es muy frecuente que sobre éstos se presente el análisis factorial como parte del análisis de las evidencias de validez de constructo (Pérez-Gil, Chacón-Moscoso y Moreno-Rodríguez, 2000), aunque también se encuentran críticas a esta manera de proceder (Batista-Foguet, Coenders y Alonso, 2004; Carretero-Dios y Pérez, 2007; Nunnally y Bernstein, 1995). Sin embargo, ni en los trabajos sobre escalas originales anglosajonas, ni en los de nuestro entorno cultural y lingüístico suelen encontrarse ejemplos donde aparezca una definición explícita de la variable, ni las fases que llevaron a los autores hasta la definición operacional correspondiente. Estos pasos son de suma importancia ya que posibilitan un acercamiento ordenado desde la definición conceptual hasta la elección de las conductas (ítems) que la ponen de manifiesto. Todo este proceder culmina con la propuesta, por parte del autor del instrumento de medida, de unas tablas de especificaciones del test (definición semántica del constructo) y de los ítems que tratan de representarla, que permitirán no sólo una valoración fundamentada de lo que el autor del instrumento quiere realmente medir, sino de la calidad y la representativi-

* Dirección para correspondencia [Correspondence address]: Macarena de los Santos-Roig. Facultad de Psicología. Campus Cartuja. C.P. 18071. Granada (España). E-mail: dlsantos@ugr.es
Title: From semantic definition to items through test's and items `specifications: construction of ERCE Scale.

Abstract: The aim of this work is to stress the relevance of all the previous steps before items construction (semantic definition, test and items specifications, experts judges, etc.). There are few works in attitude tests construction where all the steps before items where showed, despite its importance is remarked by standards (AERA, APA, NCME, 1999). The process of ERCE Scale construction is showed as an example in that way. ERCE (De los Santos-Roig, 2009) was developed to assess illness representations which is a construct inserted in Common Sense Model of Illness (Leventhal et al, 1997). In the second part of this work qualitative items analysis of scale is showed and discussed.

Key words: Illness Representation; semantic definition; test and items specifications; items construction; experts judges; content validity; items analysis; ERCE Scale.

dad de los ítems que finalmente la conforman. Generalmente esto no se muestra (Smith, Fisher y Fister, 2003), a pesar de ser considerado como fundamental en los estándares (AERA et al., 1999) y de que existen numerosos trabajos donde se justifica sobradamente su importancia (Carretero-Dios y Pérez, 2005; Muñiz y Fonseca-Pedrero, 2008; Osterlind, 1989; Rubio, Berg-Weger, Tebb, Lee y Rauch, 2003). Las especificaciones quedan avaladas por las decisiones finales que los distintos autores adoptan. Sin embargo, cuando en alguna ocasión estas fases se hacen explícitas son muchas las posibilidades que se ofrecen a la hora de interpretar y criticar las decisiones hasta la propuesta de dichas tablas de especificaciones. Un buen ejemplo de ello lo ofrecen Carretero-Dios, Pérez-Meléndez y Buela-Casal (2009).

Por otro lado, es cierto, que la presentación de la definición semántica de un constructo y de las decisiones hasta su concreción difícilmente tienen cabida en un artículo, dadas las limitaciones a las que este formato está sometido, pero sí que se puede hacer un intento por mostrar resumidamente qué se hizo antes de llegar a establecer los ítems y porqué, o al menos ofrecer la tabla de especificaciones de la que se parte para el desarrollo de los ítems de la prueba en cuestión.

El constructo Representación de la Enfermedad forma parte del Modelo de Autorregulación desarrollado por Leventhal (véase, por ejemplo, Leventhal et al., 1997). Está formado por dos componentes: una representación cognitiva (RCE) y una emocional (REE). Varios instrumentos han sido desarrollados para la medición del constructo; entre otros, el Implicit Models of Illness Questionnaire (Turk, Rudy y Salovey, 1983) y el Illness Perception Questionnaire, IPQ (Moss-Morris, Weinman, Petrie, Horne, Cameron y Buick, 2002; Weinman, Petrie, MossMorris y Horne, 1996). Actualmente, el IPQ-Revised (MossMorris et al., 2002) es el instrumento que más se utiliza. Ha sido aplicado a un gran número de enfermedades y traducido a multitud de idiomas (Hagger y Orbell, 2003; Quiceno y Vinaccia, 2010), entre ellos al español. En España, existen las 
adaptaciones del IPQ-R-Versión Española, (Beléndez-Vázquez, Bermejo-Alegría y García-Ayala, 2005), y del Cuestionario de Creencias sobre la Enfermedad, CCRE, (Van der Hofstadt y Rodríguez-Marín, 1997) que es como denominaron los autores a la versión española del IMIQ. Sin embargo, estas adaptaciones presentan algunas carencias. Algunas tienen que ver con la intencionalidad del instrumento y con el tipo de muestra que se ha utilizado; en el CCRE personas sanas (estudiantes y enfermeros) que contestan sobre diversas enfermedades. En el caso del IPQ-R-Versión Española, las autoras realizaron una traducción del original y la administraron a una muestra de hipertensos. En su trabajo de 2005, aparecen los resultados del análisis factorial, algunos datos de fiabilidad y no se aportan más evidencias de validez que los resultados para sexo y edad, por lo que la adaptación aún no puede considerarse definitiva.

Por otro lado, en los últimos años, los mismos autores del IPQ se han planteado la necesidad de ir más allá y de considerar seriamente las limitaciones del instrumento (French y Weinman, 2008). Entre otras, se comentan la incapacidad para evaluar la representación específica para una enfermedad, la dificultad de cara a la intervención para interpretar algunas sub-escalas, que son ambiguas en algunas enfermedades como la diabetes $(v \cdot g$. control de la enfermedad), $y$, en definitiva, la imposibilidad de medir en profundidad y con mayor sensibilidad las creencias de los pacientes.

Todo lo comentado sobre los instrumentos disponibles en nuestro ámbito cultural, así como las carencias de los instrumentos desarrollados en otros idiomas, llevaron a plantear la necesidad de construir una escala en español, y de ahí surgió el trabajo que actualmente venimos realizando con la escala ERCE (De los Santos-Roig, 2009). La ERCE ha sido desarrollada, hasta el momento, para medir los cinco componentes del "bloque estructural básico" (Heijmans y De Ridder, 1998) de la Representación Cognitiva. No se ha incluido aún (lo que no indica que no se vaya a considerar en futuros trabajos) ni la coherencia, ni el curso cíclico, ni la Representación Emocional, como en otros instrumentos. La razón es muy sencilla; las citadas dimensiones requieren todavía, a nuestro juicio, una profundización teórica, una definición y una concreción de sus relaciones con otros constructos que hasta ahora no se ha realizado. Es fundamental como paso inicial para la medición el concretar la definición semántica y sintáctica, y abordar rigurosamente la medida de estos componentes requiere un trabajo profundo y específico sobre ello. Lo que se comenta se ha hecho con la Representación Cognitiva; incluir también las demás dimensiones en este trabajo hubiera sobrepasado los límites de nuestra capacidad.
Como hemos dicho, este trabajo se centra en la Representación Cognitiva de la Enfermedad (RCE) cuya estructura quedó configurada por las creencias relativas en torno a cinco componentes. Estos hacen referencia a la identidad de la enfermedad (identificación de los síntomas que la caracterizan), las causas que la provocan, las consecuencias que tiene, el control que puede ejercerse sobre ésta y el curso esperado a través del tiempo.

El objetivo de este trabajo es, por un lado, mostrar con un ejemplo las fases fundamentales en la construcción de una escala (desde la definición conceptual hasta la propuesta de las tablas de especificaciones de todas las sub-escalas que finalmente conformaron la ERCE), y por otro presentar esta nueva medida en español del constructo RCE. Como considerar en un único artículo los ítems desarrollados, junto con los juicios y el análisis de ítems tradicional alargaría innecesariamente la extensión de este trabajo, se mostrarán las especificaciones del test y de los ítems, la construcción de los mismos y sólo los resultados del análisis de ítems cualitativo. Es decir, se hará una valoración de lo obtenido en los ensayos preliminares con la prueba, así como del juicio de expertos sobre los ítems, dejando para otro trabajo la presentación del análisis de ítems cuantitativo (frecuencias, descriptivos básicos, homogeneidad, discriminación, etc.), así como resultados sobre la fiabilidad y las evidencias de validez. Al final, en el Anexo, se presenta la escala que fue elaborada.

\section{Delimitación del constructo y su concreción en tablas de especificaciones}

\section{La tabla de especificaciones del test}

La tabla de especificaciones del test incluye la información relativa a los ítems que se desarrollaron una vez se tuvo la definición semántica y fue explicitada la importancia o peso que cada sub-componente tendría en el total de su respectiva escala. Téngase en cuenta que la escala ERCE está compuesta por un total de cinco escalas independientes entre sí, aunque relacionadas, que de momento no ofrecen una puntuación total. Por ello, cuando nos referimos a escalas, nos estamos refiriendo a un componente concreto y a los distintos sub-componentes que han sido considerados en su definición.

En la Tabla 1 aparecen representados y definidos los sub-componentes de los cinco aspectos de la RCE que evalúa la ERCE: identidad, causas, consecuencias, control/cura y curso, así como la importancia que se otorgó a los diversos aspectos incluidos. 
Tabla 1. Tabla general de Especificaciones del Test.

\section{COMPONENTES y FACETAS}

IDENTIDAD: Esta dimensión incluye dos tipos de información: percepción de los síntomas, creencias sobre la etiqueta que puede representar ese conjunto de síntomas y creencias sobre las asociaciones entre los síntomas y la etiqueta de la enfermedad

- SÍNTOMAS PERCIBIDOS: Listado de síntomas pertenecientes o no a la enfermedad $\mathbf{5 0 \%}$

- ENLACE ETIQUETA-SÍNTOMAS: Síntomas que se cree tienen que ver con ésta $\mathbf{5 0 \%}$

CAUSAS: Se refiere a las creencias individuales sobre los factores (externos e internos) que son responsables de causar la enfermedad u otros aspectos de ésta (p.e. los síntomas)

- Medioambiental: Se refiere a cualquier causa presente en el medio exterior al paciente (p.e. contaminación) $20 \%$

- Biológica: Causas que tienen que ver con aspectos relativos al desarrollo o a la evolución física o biológica de la persona (p.e. anomalías físicas) $20 \%$

- Conductual: Causas relativas al estilo de vida, a los hábitos, o a la manera de hacer determinadas cosas en la vida cotidiana (p.e. comida basura) $20 \%$

- Psicológica/Psico-Social: Causas relativas a los aspectos psicológicos (como la forma de afrontar la vida) o al entorno social en que se desarrolla la persona (p.e. las relaciones con los demás) $20 \%$

- Mística: Hacen referencia a todas aquellas causas inexplicables o explicadas por razones superiores al hombre (p.e. causas sobrenaturales) $20 \%$

CONSECUENCIAS: Dimensión que se compone de aspectos relativos a las consecuencias actuales o futuras que la enfermedad o algunos aspectos de éstas tienen o tendrán sobre distintas áreas de la vida del paciente (laboral, familiar, de pareja, etc.)

ACTUALES: Se refiere a las consecuencias que esté teniendo la enfermedad o aspectos relativos a ella sobre distintas áreas de la vida de la persona, en este momento, a día de hoy. $\mathbf{5 0 \%}$

- De los síntomas: Familiares, Sociales, Laborales, Personales

- Del curso: Idem

- Del control: Idem

ESPERADAS: Se refiere a las expectativas de los enfermos, es decir, a las consecuencias que creen que la enfermedad tendrá en un futuro, a corto, medio o largo plazo. $50 \%$

- De la enfermedad: Idem

CONTROL/CURA: Dimensión compuesta por aspectos relativos a las creencias de control en general, sobre la enfermedad o sobre algún aspecto de ésta y de la posibilidad de curación. También las creencias de control pero relativas al agente que lo lleva a cabo, es decir, en manos de quién está el control (propio paciente, familiares, tratamiento, médicos).

CONTROL PERCIBIDO: Se refiere a las creencias generales de control, sin especificar en manos de quién está esa posibilidad de controlar (p.e. es posible controlar mi enfermedad) $20 \%$

- Sobre la enfermedad, síntomas.

CONTROL PERSONAL: Se refiere a las creencias sobre la posibilidad de que las propias conductas o uno mismo sea el agente de control de su propia enfermedad (p.e. yo controlo mi enfermedad) $20 \%$

- Sobre la enfermedad, síntomas, consecuencias y causas

CONTROL DEL TRATAMIENTO: Se refiere a creencias sobre el papel del tratamiento en el control de la enfermedad (p.e. el tratamiento controla la enfermedad) $20 \%$

- Sobre los síntomas, causas y las consecuencias

CONTROL DE OTROS SIGNIFICATIVOS: Se refiere a la creencia en si el control de la enfermedad está en manos de otras personas importantes para el paciente (el médico o la familia) $20 \%$

- Sobre los síntomas y las causas

CURA: Se refiere a creencias generales sobre la posibilidad de curación de la enfermedad o de aspectos relacionados con ella (p.e. la enfermedad es incurable) $20 \%$

- De la enfermedad

CURSO: Compuesto por aspectos relativos a la duración/curso o a la evolución de la enfermedad o de algún aspecto de ésta

DURACIÓN: Hace referencia a creencias sobre aspectos que tienen que ver con las creencias sobre la duración de la enfermedad o el curso de ésta (p.e. sobre la cronicidad de la enfermedad) $\mathbf{5 0 \%}$

EVOLUCIÓN: Se refiere a creencias sobre cómo va a evolucionar la enfermedad con el paso del tiempo $\mathbf{5 0 \%}$

Como puede observarse la importancia dada a las distintas facetas aparece junto a la definición en un recuadro y, como no existen a priori razones teóricas suficientes como para establecer pesos diferentes por faceta, éstos fueron repartidos por igual dentro de cada componente.

\section{La tabla de especificaciones de los ítems}

El proceso de construcción de ítems tiene como objetivo desarrollar los elementos que servirán para hacer operacionales los componentes a los que se ha hecho alusión en la ta- 
bla de especificaciones del test. Si bien es cierto que éste es uno de los aspectos a los que menos atención se dedica dentro de la psicometría (Prieto y Delgado, 1996), su relevancia es fundamental ya que ítems no representativos de los componentes o inadecuadamente redactados llevarán a problemas posteriores que impedirán la consecución de una prueba de calidad. Los ítems suponen la operacionalización de las definiciones a través de las que el constructo o los constructos son materializados, así que la importancia de desarrollarlos adecuadamente es evidente.
Al iniciar la construcción de los ítems es necesaria la especificación de las características que éstos han de tener para que el constructor de los mismos los concrete de manera adecuada. Así, es fundamental que se clarifique qué objetivo tiene la escala, qué tipo de ítems se van a plantear, qué formato van a tener, qué forma de puntuación, qué tipo de instrucciones podrían desarrollarse, etc.

Todo esto quedó recogido en tabla de Especificaciones de los Items (Tabla 2), que fue construida según recomendaciones de Osterlind (1989), y donde se puede observar toda la información que guió el proceso de elaboración.

Tabla 2. Tabla de Especificaciones de los Ítems.

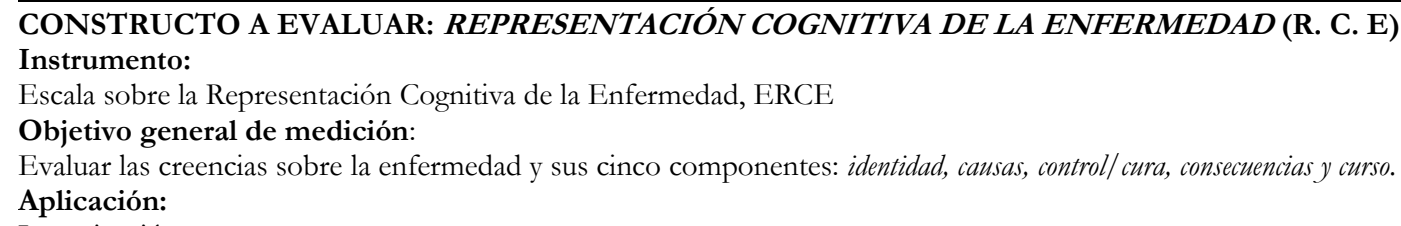

- Evaluar los síntomas experimentados por el paciente que éste relaciona/identifica como parte de la enfermedad

Tareas:

- El evaluado deberá indicar los síntomas que padece o que están presentes en su vida cotidiana.

- Deberá distinguir cuáles de ellos son representativos o forman parte de la propia identidad de la enfermedad, indicando su relación con ésta.

\section{ATRIBUTOS DEL ÍTEM}

Formato: Listado y pregunta directa

Contenido: Síntomas y pregunta en cada síntoma sobre su relación con la enfermedad

\section{ATRIBUTOS DE LAS RESPUESTAS:}

Formato de la Escala de respuesta: Dos escalas dicotómicas; una para marcar una casilla en caso de "presencia" y otra con formato Sí/No (Marcar con una cruz la presencia del síntoma y en caso de presencia, señalar si se enlaza o no con la enfermedad)

Proporción de ítems dentro de la escala: 50\% (síntomas) 50\% (preguntas sobre la relación del síntoma con la enfermedad)

\section{Ejemplo de instrucción:}

"A continuación aparece una lista con una serie de sintomas. Queremos que nos indiques cuáles de ellos padeces ACTUALMENTE, EN TU DIA A DLA.

Para ello, marca con una cruz la casilla que aparece junto a cada uno. Posteriormente, sobre cada sintoma marcado, queremos que nos indiques si crees que está relacionado o no con tu enfermedad. Si tienes alguna duda, levanta la mano y te atenderé de inmediato."

\section{Ejemplo de ítem:}

1. Nauseas $\square$ ¿Crees que este síntoma tiene algo que ver con tu enfermedad? Si No

\begin{tabular}{l}
\hline Componente 2: CAUSAS \\
\hline Objetivo: \\
- Evaluar las causas de diversas tipologías causales (medioambientales, psicosociales, místicas, etc.) que el paciente atribuye al origen de \\
su enfermedad o a aspectos relacionados con ésta (los síntomas, por ejemplo). \\
Tareas: \\
- El evaluado deberá indicar a qué causa o causas cree que se debe el origen de su enfermedad \\
- Idem para los síntomas: deberá indicar a cuál o cuáles de las causas atribuye los síntomas de su enfermedad \\
\hline ATRIBUTOS DEL ÍTEM \\
Formato ítem: Listado de posibles causas para marcar si se atribuye al origen o a los síntomas de la enfermedad \\
Contenido: Causas de diversas tipologías; medioambientales (p.ej. polución), biológicas (p.ej. un virus), conductuales (p.ej.estrés), psicosociales (p. ej. \\
Atención médica), místicas (p.ej. azar), etc.
\end{tabular}

\section{ATRIBUTOS DE LAS RESPUESTAS:}

Formato de la Escala de respuesta: Una escala dicotómica en forma de una casilla para marcar en caso de que se atribuya esa causa al origen de la enfermedad o a la aparición de los síntomas.

Proporción de ítems dentro de la escala: 50\% causas relativas al origen, 50\% causas relativas a los síntomas 


\begin{abstract}
Ejemplo de instrucción:
"Como sabes, las enfermedades pueden estar causadas por diversos factores o situaciones. Abora queremos que nos indiques, de todas estas causas cuáles crees que ban favorecido que tu enfermedad se desarrollara en un principio, y cuáles de ellas pueden, a tu juicio, provocar algunos de tus sintomas diarios. Lo que tienes que hacer es leer este listado de causas e indicar aquellas que creas que estuvieron en el origen de tu enfermedad, y que están en la aparición de los sintomas cotidianos. Para ello, junto a cada causa aparecerá una casilla donde la inicial E, bará referencia a la enfermedad, es decir, a si crees que el origen de la enfermedad se debió a ese factor, y $S$ a si crees que esa es una de las causas de los sintomas que padeces diariamente. En caso de que creas que una misma causa ha provocado la enfermedad y actualmente provoca los sintomas al mismo tiempo, marca las dos opciones. Si crees que esa causa NO es responsable de ninguna de las dos, déjala sin marcar y pasa a la siguiente. Si tienes alguna duda, levanta la mano y te atenderé de inmediato."
\end{abstract}

Ejemplo de ítem:

Tabaco

E $\mathrm{S}$

Componente 3: CONSECUENCIAS

Objetivo:

- Evaluar las consecuencias de la enfermedad en diversas áreas de la vida de la persona (familiar, social, etc.), tanto actuales como esperadas (a medio o largo plazo).

Tarea:

- El evaluado deberá contestar sobre el grado en que la enfermedad afecta a distintas áreas de su vida, tanto en el momento actual como lo que percibe que pasará en un futuro.

\title{
ATRIBUTOS DEL ÍTEM
}

Formato ítem: Preguntas directas

Contenido: Preguntas relativas a las consecuencias (actuales y futuras) de la enfermedad o de algunos aspectos de ésta (en concreto, las consecuencias de los síntomas, el curso y el control) en diversas áreas de la vida de la persona (familia, pareja, trabajo, etc.)

\section{ATRIBUTOS DE LAS RESPUESTAS}

Formato de la Escala de respuesta: Escala tipo Likert de 5 puntos (1 No afecta Nada - 5 Afecta Mucho)

Proporción de ítems dentro de la escala: 50\% para las actuales, 50\% para las futuras.

Instrucciones: Ejemplo de instrucciones sobre los ítems relativos a las consecuencias actuales de los sintomas.

"Nos interesa saber cuáles son las consecuencias que la enfermedad tiene sobre ti y para ello vamos a centrarnos en TUS SÍNTOMAS. Queremos saber en qué grado te afectan. Para ello se te van a presentar una serie de preguntas que deberás responder, rodeando con un círculo un número entre el 1 y el 5 , de manera que 5 significaria que los sintomas te afectan mucho y 1 que no te afectan nada. Siguiendo estas instrucciones, lee las siguientes preguntas y marca tu respuesta. Por favor, no dejes ninguna pregunta sin contestar."

Ejemplos de ítems:

De 1 a 5, ¿cómo afectan los síntomas de tu enfermedad a tu propia familia?

\section{Objetivos:}

1. Evaluar las creencias sobre el control que se percibe de la enfermedad o de aspectos relacionados con ésta.

2. Evaluar las creencias sobre la posibilidad de que la enfermedad se cure.

Tareas:

1. El evaluado deberá contestar sobre el grado de control que tiene sobre la enfermedad, a través de él mismo o de otros agentes (otros, la medicación, etc.)

2. Deberá contestar sobre su creencia acerca de la posibilidad de que su enfermedad llegue a curarse.

\section{ATRIBUTOS DEL ITEM}

Formato ítem: Preguntas directas

Contenido: Frases relativas a las creencias de control o cura sobre la enfermedad o sobre aspectos relacionados con ésta. Los ítems se refieren a creencias sobre la cura y sobre el control en general y también centrado en el agente que lo lleva a cabo: uno mismo, el médico, el tratamiento, etc.

\section{ATRIBUTOS DE LA RESPUESTA}

Formato de la Escala de respuesta: Escala tipo Likert de 5 puntos (1 Nada controlable - 5 Muy controlable)

Proporción de ítems dentro de la escala: 20\% para el control general, 20\% para el control personal, 20\% para control del tratamiento, $20 \%$ para control de otros importantes, $20 \%$ para la cura.

\section{Ejemplo de instrucción:}

"Antes te hemos preguntado por las consecuencias que tiene para ti tu enfermedad. Ahora, queremos que nos indiques lo controlable que te parece que es tu enfermedad, es decir, si crees que existen estrategias que permitan o que hagan posible mantenerla bajo control, estable, manejada, etc. Para indicar todo esto se te van a presentar una serie de preguntas, a las que deberás responder rodeando con un círculo un número del 1 al 5 igual que en ocasiones anteriores (sabiendo que 5 significa que es muy controlable y 1 que no es nada controlable)".

Ejemplo de ítem:

De 1 a 5, ¿en qué grado crees que tu enfermedad es controlable? 


\section{Componente 5: CURSO}

Objetivo: Evaluar las creencias sobre el curso (evolución y duración) de la enfermedad.

Tarea: El evaluado deberá contestar acerca de su creencia sobre la duración y la evolución que va a tener la enfermedad

\section{ATRIBUTOS DEL ITEM}

Formato ítem: Afirmaciones o frases afirmativas

Contenido: Afirmaciones relativas al curso de la enfermedad, o de aspectos relacionados con ella.

\section{ATRIBUTOS DE LA RESPUESTA}

Formato de la Escala de respuesta: Alternativas de respuesta para marcar la que se considere más acorde con la opinión del evaluado.

Proporción de ítems dentro de la escala: 50\% para la evolución, 50\% para la duración.

\section{Ejemplo de instrucciones:}

"Nos interesa que nos digas lo que piensas sobre la duración de tu enfermedad, es decir, nos interesa conocer si crees que va a durar poco, o si crees que estará presente durante mucho tiempo. Para que nos lo indiques, te vamos a presentar tres frases. Deberás decirnos cuál de las tres representa mejor tu opinión marcando con una cruz SOLO UNA DE ELLAS.”

Ejemplo de ítem:

Mi enfermedad estará siempre presente

\section{Construcción y análisis cualitativo de los ítems}

La versión piloto de la escala: primeros ítems y su análisis cualitativo

Es necesario advertir que, aunque las especificaciones de los ítems se refieran a aquellos relativos a cualquier enfermedad, finalmente las instrucciones y los ítems desarrollados en la versión piloto de la ERCE se centraron en la Diabetes Mellitus; una enfermedad representativa de las enfermedades crónicas más comunes en nuestra sociedad, con un gran componente "conductual" y que depende en gran medida de la auto-regulación del paciente. Se trata en definitiva de una "enfermedad prototipo" que puede encajar muy bien en el modelo teórico que guía todo este trabajo. Ello no quiere decir que el objetivo final de la prueba abandone la posibilidad de ser aplicable a cualquier enfermedad, aunque para ello aún deban darse algunos pasos. En el Anexo de este trabajo aparece la versión definitiva para todas las sub-escalas de la ERCE.

\section{Construcción de ítems}

Los ítems se desarrollaron para cada componente, teniendo en cuenta su definición.

\section{Componente "Tdentidad"}

Partiendo de la definición semántica de la identidad ésta se refiere por un lado, a los síntomas percibidos por el paciente (en su vida diaria) y, por otro, al enlace que hace de éstos con la enfermedad que padece (es decir, la identidad debe recoger la opinión o la creencia sobre qué síntoma o síntomas están relacionados con la enfermedad). Los ítems desarrollados para representar este componente debieran atender a las dos cuestiones planteadas. Por esto, se desarrolló inicialmente un listado con 33 síntomas comunes y representativos de la Diabetes Mellitus, así como una pre- gunta directa, para ser contestada en caso de que se padezca, sobre su relación con la etiqueta "diabetes".

Este listado fue elaborado teniendo en cuenta diversas fuentes de información (libros, otras escalas de evaluación, consulta a expertos, etc.) y se desarrolló con la instrucción concreta de que la persona que contestara la escala, indicara si en su día a día padecía alguno de los síntomas del listado. Sin embargo, lo que finalmente interesaba evaluar no era la presencia/ausencia del síntoma (se trataba de una escala dicotómica SI/NO), sino si la persona lo relacionaba con la etiqueta de la enfermedad. Por ello se añadió una pregunta que sondeaba la creencia sobre si el síntoma concreto estaba relacionado o no con la misma.

\section{Componente "Causal"}

Para el caso de este componente, y considerando que la definición semántica hacía referencia a diversos tipos de causas posibles, se desarrollaron 28 ítems referidos a causas medioambientales, biológicas, conductuales, psicológicas/psicosociales y místicas. Por ello, en un primer momento se tuvieron en cuenta tanto aquellas causas consideradas en el estudio de Shiloh, Rashuk-Rosenthal y Benyamini (2002), como las que aparecían en la bibliografía relativa a la Representación de la Enfermedad en general, y se pusieron ejemplos representativos de cada una de las categorías. Para las conductuales y psicológicas/psicosociales, debido a la diversidad de conductas que podrían representarlas, se listaron un número significativamente mayor de causas posibles que para el resto.

También se tuvo en cuenta un hecho importante que había sido contemplado en la definición semántica; las causas podrían atribuirse tanto al origen de la enfermedad, como a los desencadenantes de la aparición de los síntomas derivados de ésta. Así, se diferenciaron, en cada caso, dando al evaluado la instrucción de que debía contestar primero pensando en el origen de la enfermedad, y luego en esas mismas causas pero como desencadenantes de los síntomas cotidianos. Considerar esta distinción es una aportación de nuestro trabajo ya que no ha sido tenida en cuenta en otros instru- 
mentos. Añade información específica para la representación de la enfermedad (en el sentido que antes comentábamos de French y Weinman, 2008), y ha mostrado evidencias por sus relaciones con otros constructos como afrontamiento o el ajuste del paciente (De los Santos-Roig, Pérez, RuízGonzález, Guardia-Archilla y Martínez-García, 2010).

Tras establecer la tabla de especificaciones de los ítems, donde se muestran ya algunos ejemplos de éstos así como de las instrucciones, se desarrollaron todos los elementos que compusieron la versión piloto de la prueba. Ésta fue sometida a diversas pruebas (juicio por parte de pacientes y análisis cuantitativo de ítems).

\section{Componente "Consecuencias"}

Para el desarrollo de los ítems de la escala de consecuencias, se tuvieron en cuenta los aspectos recogidos en la definición de la Tabla 1. Obsérvese que las consecuencias se dividen en actuales y esperadas, y que dentro de ambas definiciones se recogen aspectos relativos a diversos componentes de la enfermedad y a distintas áreas de la vida. Esta definición fue resultado de una profundización teórica y empírica que sirvió para poner en común las definiciones dadas también por otros autores (Cameron, Leventhal y Leventhal, 1993; Diefenbach y Leventhal, 1996; Leventhal, Brisette y Leventhal, 2003; Scharloo y Kaptein, 1997). En total fueron construidos 16 ítems para recoger todos los aspectos incluidos en la definición.

\section{Componente "Control/Cura"}

En la definición de esta dimensión (Tabla 1) se enfatizaron tanto los distintos agentes de control (Skinner, 1996; Walker, 2001), como los fines del control (control sobre la enfermedad, sobre los sintomas, las consecuencias, el curso y las causas), de ahí que los ítems desarrollados atendieran a estas dos características. La necesidad de considerar los fines de control también en la definición, surgió de la profundización teórica y del estudio de los instrumentos disponibles, donde sólo se consideraba el control sobre la enfermedad, sin distinguir aspectos diferentes dentro de la misma. Con esta aportación, que permite la medida del control percibido de un modo más específico y que como se ha dicho no se ha tenido en cuenta en otros instrumentos, se concretaron un total de 11 ítems. Estos representan tanto creencias relacionadas con el control percibido (sobre la enfermedad, y sobre los aspectos relacionados con ésta) como con las relativas a los agentes de control: el personal, el de otras personas importantes en la vida de la persona o el del tratamiento. Finalmente, se desarrolló un ítem relacionado con la cura que fue considerada también, al igual que en la definición original de Lau y Hartman (1983), como parte de este componente. Dicho ítem sondea la creencia sobre la posibilidad de curación de la enfermedad.

\section{Componente "Curso"}

En lo relativo a este componente, para la redacción de los ítems se tuvo en cuenta que se distinguía entre la evolución y la duración, tal y como aparece en la Tabla 1. Para evaluar estos aspectos se desarrollaron dos ítems, con tres y cuatro alternativas de respuesta, respectivamente, como queda contemplado en el Anexo.

\section{Análisis de ítems cualitativo: el juicio de expertos y los ensayos preliminares}

Una vez desarrollados los primeros ítems, éstos se sometieron a una serie de "pruebas" entre las que se encuentra el juicio de expertos sobre los ítems. El análisis de ítems tradicionalmente ha sido enfocado hacia el "comportamiento" estadístico de los mismos, pero en este trabajo, siguiendo algunas recomendaciones de Osterlind (1989), se prestó una atención especial también al análisis cualitativo, volviendo de este modo a considerar la definición semántica. Para ello se utilizaron, por un lado, las valoraciones realizadas por una serie de jueces sobre la relevancia de los ítems para el constructo, tal y como había sido definido (juicio de expertos sobre los ítems), y por otro se analizaron los resultados de los ensayos preliminares de la prueba

\section{El juicio de expertos: Los primeros items y la evaluación de la pertenencia a los componentes de la RCE.}

El juicio de expertos es un procedimiento fundamental en la creación de una prueba, que se enmarcaría dentro de la validez de contenido. Aunque es la validez de constructo la que se refiere al grado en que un instrumento de medida evalúa el constructo objetivo (Haynes, Richard y Kubany, 1995), dentro de ésta podríamos hablar de distintas fuentes de validez entre las que se incluye a la validez de contenido. Ésta se define como aquella referida al grado en que el constructo está adecuadamente definido y representado a partir de los items del instrumento de medida (Haynes et al., 1995), o lo que es lo mismo, el grado en que los ítems son pertinentes para el constructo en sí, tal y como éste ha sido definido. Por otro lado, este proceso de obtención de evidencias de validez de contenido puede servir para refinar el constructo original (Smith y McCarthy, 1995), lo que indica su importancia más allá de la propia construcción de la prueba.

Diversos aspectos de la validez de contenido, como el juicio de la definición semántica o el juicio sobre los items, son aspectos centrales en la construcción de la prueba. Tanto uno como otro forman parte de lo que se denomina validez de contenido y, aunque éste no fue un estudio de validez propiamente dicho, sus resultados tuvieron un peso importantísimo en el proceso de construcción de la prueba.

Una vez desarrollados los ítems, se procedió a verificar su "pertenencia" al componente de referencia según la definición semántica. Para ello, se pidió colaboración a una serie profesores e investigadores de las áreas de Metodología 
de las Ciencias del Comportamiento, Psicología Clínica y Psicología de la Salud de distintas universidades de España, bien personalmente, bien a través de correo electrónico. Su tarea consistió en clasificar cada uno de los ítems en el componente o los componentes que creyeran oportunos. En total se habían desarrollado 30 ítems, que fueron repartidos en dos cuadernillos conformados al azar. Esto llevó a que cada juez evaluara sólo la mitad de los elementos. Del total de jueces a los que se les pidió participar, contestaron aproximadamente la mitad. Para cada uno de los cuadernillos se obtuvieron 7 y 8 juicios, respectivamente. Por razones obvias de espacio, no se van a incluir los cuadernillos en este artículo (en caso de interés, consultar De los Santos-Roig, 2009).
Los resultados sobre la consulta indicaron que en la mayoría de los casos más del 75\% de los jueces opinaron que el ítem pertenecía al componente para el que se había desarrollado, si bien algunos ítems presentaban un porcentaje más bajo; entre el $50 \%$ y el $62.5 \%$ de acuerdo (Tabla 3). Analizando descriptivamente estos resultados se observó que en muchos de los casos los jueces habían marcado dos opciones como posibles. En las instrucciones, se indicó a los jueces que marcaran el componente/los componentes al que creían que pertenecía el ítem. Esto se hizo con el único objetivo de no limitar las respuestas a priori y comprobar si la mayor parte de los ítems se categorizaban en los componentes definidos (véase De los Santos-Roig, 2009).

Tabla 3. Resultados del Juicio de Expertos en porcentajes (aparecen sólo los ítems del Cuadernillo 2 que no han obtenido como mínimo el $75 \%$ de acuerdo).

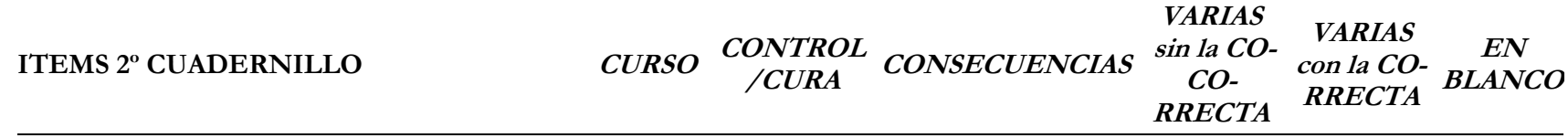

Piensa en tu trabajo u obligaciones diarias sean éstas como sean, es decir, remuneradas o no, domésticas, laborales, etc. ¿Cómo te afectan los síntomas de la enfermedad?

$25 \% \quad 12.5 \% \quad \mathbf{6 2 . 5 \%}$

Contesta escogiendo la frase que mejor represente tu opinión:

a. Mi enfermedad se mantendrá siempre como hasta ahor

b. Mi enfermedad empeorará progresivamente

c. Mi enfermedad mejorará con el tiempo

d. Mi enfermedad irá cambiando por temporadas

Piensa en tu trabajo u obligaciones diarias sean éstas como sean, es decir, remuneradas o no, domésticas, laborales, etc. ¿Cómo te afecta la evolución diaria de la enfermedad?

$\begin{array}{llll}12.5 \% & \mathbf{5 0} \% & 12.5 \%\end{array}$

Contesta escogiendo la frase que mejor represente tu opinión:

a. Mi enfermedad estará siempre presente

\begin{tabular}{|c|c|c|c|c|c|}
\hline $\begin{array}{l}\text { ¿En qué grado afecta la evolución diaria de tu en- } \\
\text { fermedad a tu familia? }\end{array}$ & $12.5 \%$ & $12.5 \%$ & $62.5 \%$ & $12.5 \%$ & \\
\hline $\begin{array}{l}\text { ¿Cómo te afecta todo lo que necesitas hacer por tu } \\
\text { enfermedad personalmente, es decir, a ti mismo } \\
\text { como persona; en tu estado de ánimo, en tu mane- } \\
\text { ra de ver la vida, en tus proyectos, etc.? }\end{array}$ & & $12.5 \%$ & $50 \%$ & $25 \%$ & $12.5 \%$ \\
\hline $\begin{array}{l}\text { ¿En qué grado afecta a tu familia todo lo que tie- } \\
\text { nes que hacer para controlar tu enfermedad? }\end{array}$ & & $12.5 \%$ & $62.5 \%$ & $25 \%$ & \\
\hline
\end{tabular}
nes que hacer para controlar tu enfermedad?

En negrita aparece el componente donde se enmarcaría el ítem, según la definición.

El trabajo de los jueces en el proceso de desarrollo de una escala es siempre una cuestión voluntaria, que requiere tiempo y esfuerzo y que la mayor parte de las veces no se ve suficientemente recompensada, ni justificada. Precisamente por ello, se puede entender que a veces la tarea se haga con desinterés o con celeridad. Con ello no se pretende en absoluto menospreciar el trabajo que realizan los jueces, aunque sí remarcar las condiciones, en las que muchas veces, se lleva a cabo este tipo de tareas. Por tanto, sabiendo que el propio proceso del juicio de expertos tiene estas características, y analizando las respuestas que se han dado a algunos de los ítems se puede apuntar a que es posible que razones como no prestar suficiente atención, no leer con detenimiento, etc., pueden estar a la base de muchas de las respuestas (y de que se clasifique a un ítem como relativo a un componente que semánticamente está muy alejado de él). Por ejemplo, en la 
Tabla 3 llaman la atención los resultados que se han obtenido en relación con el ítem - "Piensa en tu trabajo u obligaciones diarias sean éstas como sean, es decir, remuneradas o no, domésticas, laborales, etc. ¿Cómo te afecta la evolución diaria de la enfermedad?”-, ya que algunos de los expertos han contestado que se trataba de un ítem relativo al curso de la enfermedad o al control. De hecho, si se lee la pregunta con detenimiento, se podrá observar que en realidad, formaría parte de las consecuencias de la enfermedad, no del curso como pudiera pensarse con un rápido vistazo al leer las palabras evolución y diaria en el ítem.

Además de las razones dadas anteriormente que pueden implicar rapidez o superficialidad a la hora de realizar la tarea, no se quiere acabar sin enfatizar que se trata de un problema no sólo de los jueces, sino relativo a todo el proceder en esta etapa; desde las instrucciones que se dan, el procedimiento a través del cual se pide a las personas que participen como jueces, las justificaciones que se ofrecen sobre su importancia, el seguimiento que se hace del trabajo, hasta el beneficio que los jueces pueden percibir por haberlo realizado, etc. Está claro que, en ocasiones, este proceder no es el adecuado.

En el caso que nos ocupa, sobre la valoración del grado de pertenencia de una serie de ítems a sus componentes, quizás algunos de los resultados puedan explicarse en parte por estas razones. Sin duda alguna, es una limitación no haber atendido con mayor rigurosidad a todos estos aspectos, ya que en caso contrario los resultados habrían sido mucho más enriquecedores y válidos. Este tipo de tareas proporcionan una información muy valiosa que contribuye a los siguientes pasos de manera fundamental.

\section{Ensayos Preliminares}

En los ensayos preliminares con un pequeño grupo de pacientes, se utilizó la Versión Piloto de la prueba, incluyendo las cinco escalas (identidad, causal, consecuencias, control/cura y curso). Es necesario decir, que el objetivo de estos ensayos no fue el de la categorización ítem-componente, sino el de comprobar si aparecían dificultades en la evaluación, debidas por ejemplo a la redacción de los ítems o de las instrucciones o a otros motivos (p.e. el haber pasado por alto aspectos importantes de la enfermedad, de los síntomas, etc.). Los comentarios de los primeros pacientes, concretamente siete voluntarios de la Asociación de Diabéticos de Granada, AGRADI, se centraron principalmente en las instrucciones, más que en los ítems en sí mismos. Se resumen en el siguiente cuadro.

Cuadro 1. Resumen de las observaciones sobre la escala en el ensayo preliminar

En la escala de identidad, aparecen las palabras "actualmente" y "en tu día a día". La idea original (partiendo de la definición semántica) era la de recoger aquella sintomatología común, frecuente, o cotidiana del paciente en su vida diaria. Sin embargo, estos términos confundían de alguna manera, ya que en estos primeros comentarios los pacientes, o bien no indicaban síntomas por entender que no eran diarios, $\mathrm{o}$ bien indicaban también síntomas que habían experimentado alguna vez, y eso se alejaba de lo que se pretendía medir. Esa instrucción fue modificada y hecha más explícita; "actualmente, en tu día a día" fue cambiado por "frecuentemente, aunque no sea diariamente". Sobre esta escala se hicieron también algunos comentarios interesantes como el de la necesidad de poner un punto intermedio “a veces” en la escala que evaluaba la creencia sobre si el síntoma pertenecía o no a la enfermedad. Al principio la escala era dicotómica (si-no), y debido a que muchos de los síntomas propios de una crisis hiper o hipo-glucémica, pueden ser parecidos a los producidos por otras razones diferentes a las de la diabetes propiamente dicha (p.e. dolor de cabeza), algunos de los participantes comentaron que no era una cuestión de "sî" o "no" para todos los casos y que en ocasiones hay síntomas presentes que son atribuidos a esta enfermedad, y que en otros casos es otra la razón de su aparición. Este matiz sobre la distinción entre distintas dolencias para los mismos síntomas, nos parece también parte de la evaluación de la identidad de la enfermedad, con lo que todos esos comentarios resultaron de un gran interés y fueron tenidos en cuenta para las modificaciones.

En el caso de la escala causal, las quejas de los participantes estuvieron centradas en la dificultad para distinguir entre las causas de los síntomas y las causas de la enfermedad, con lo que las instrucciones se aclararon más para indicar de un modo más directo la intención de lo que se pretendía recoger, a saber, la diferencia entre las causas atribuidas al origen de la enfermedad en su momento, y las atribuidas a los síntomas diarios que conlleva. También se tuvo que aclarar la intención original de la escala en cuanto a las atribuciones causales; que lo que interesaba evaluar no era el conocimiento de las posibles causas de la diabetes en general, sino su conocimiento concreto y particular, la representación causal, al fin y al cabo, de su propia diabetes.

También se realizaron comentarios sobre la necesidad de aclarar aún más las instrucciones para la escala de consecuencias en relación al curso de la enfermedad, es decir, a las consecuencias que la propia evolución diaria de ésta (con altibajos a lo largo del día) pudiera tener para la persona. Algunos participantes comentaron la dificultad de entender las palabras "desarrollo diario", "comportamiento", "evolución", y las instrucciones fueron modificadas para tratar de hacerlas más claras.

Una vez realizadas las modificaciones se llevó a cabo otro ensayo con nueve pacientes de la asociación AGRADI, donde los participantes no hicieron comentarios, por lo que no se realizaron modificaciones, aunque se obtuvo información que resultó de mucha utilidad en fases posteriores.

Finalmente y con objeto de poder comprobar si los resultados podían diferir si se utilizaba una muestra de distintas características, se realizó otro ensayo con pacientes diabéticos que provenían de un centro de salud $(n=5)$, la mayor parte de edad avanzada (>60 años) y diabéticos Tipo 2 . Este ensayo llevó a la conclusión de que el instrumento no se adaptaba bien a este tipo de pacientes, ya que las instrucciones y los ejemplos estaban dirigidos a personas más jóvenes, con un nivel educativo más alto, así como con un tipo de diabetes concreta (la Tipo 1), insulinizados y con mayor sin- 
tomatología, lo que implica que su representación esté más desarrollada. Todo esto llevó al planteamiento de que lo más viable en este caso sería continuar el trabajo con este tipo de pacientes y no con pacientes Tipo 2, ya que las implicaciones de la enfermedad son mucho más patentes, así como sus posibles relaciones con otras variables (como el afrontamiento y la salud general percibida). En definitiva, aunque el objetivo final de la prueba es que ésta sea aplicable a todo tipo de pacientes, en estas primeras fases lo más factible fue centrarse en aquella población más joven, más rica en información y con una idea de la enfermedad más elaborada. Además, no se debe olvidar que en esta fase del trabajo aún se está indagando en la definición, medida e idoneidad de la RE, por lo que es adecuado valorar los resultados sin considerar las fuentes de error no deseables que pudieran conllevar las particularidades de la muestra. Aspectos que, por el contrario, sí deben ser tenidos en cuenta en otros momentos del proceso (p.e. si se tratara de hacer una versión estandarizada de la prueba).

\section{Consideraciones finales}

Como se viene diciendo el objetivo de este trabajo era doble. Primero, se ha mostrado el proceso de construcción de una escala, partiendo de la definición semántica, concretada en la Tabla de Especificaciones del Test, así como estableciendo la guía necesaria para el desarrollo de los ítems en una Tabla de Especificaciones sobre los mismos. En ellas ha quedado plasmado cómo se han considerado algunos aspectos no incluidos en otros instrumentos adaptados en nuestro país, como son la versión española del IPQ-R (Beléndez-Vázquez et al., 2005) y el CCRE (Van der Hosftadt y Rodríguez-Marín, 1997). Así, el considerar las atribuciones causales específicas para las crisis en la diabetes, el control percibido sobre la enfermedad en general pero también específicamente sobre aspectos de ésta y la distinción entre consecuencias actuales y futuras de la enfermedad, aporta una medida del constructo más específica, sensible y útil de cara a la posible intervención (al hilo de lo que recomiendan French y Weinman, 2008). Todo ello ha sido resultado, por un lado, de la profundización teórica sobre los conceptos involucrados, nece-

\section{Referencias}

AERA, APA y NCME, (1999). Standards for educational and psychological tests. Washington DC: American Psychological Association, American Educational Research Association, National Council on Measurement in Education.

Batista-Foguet, J. M., Coenders, G. y Alonso, J. (2004). Análisis factorial confirmatorio. Su utilidad en la validación de cuestionarios relacionados con la salud. Medicina Clinica, 122 (1), 21-27.

Beléndez-Vázquez, M., Bermejo-Alegría, R. M. y García-Ayala, M. D. (2005). Estructura factorial de la versión española del Revised Illness Perception Questionnaire en una muestra de hipertensos. Psicothema, 17, 318-324.

Cameron, L. D., Leventhal, E. A. y Leventhal, H. (1993). Symptom representations and affect as determinants of care seeking in a community dwelling adult sample population. Health Psychology, 12, 171-179. saria como se decía al principio, y por otro de la revisión empírica, que ha permitido conocer las limitaciones de otros instrumentos.

Después se han comentado las valoraciones de tipo cualitativo que se realizaron tanto sobre los ítems en relación con la definición (juicio expertos), como aquellas referidas a los primeros ensayos con la prueba en pequeñas muestras. Se ha indicado cómo los resultados obtenidos para algunos de los ítems e incluso para algunas de las instrucciones podrían hacer pensar en la necesidad de realizar alguna modificación, que se ha llevado a cabo cuando se ha considerado pertinente. Se han argumentado, también, las razones para no modificar los ítems que no eran categorizados adecuadamente por los expertos, basando éstas fundamentalmente en las limitaciones de la tarea en sí misma. Está claro que los juicios sobre los ítems podrían haberse mejorado con la utilización de procedimientos como las entrevistas cognitivas (Willis, 2004), como además se viene haciendo en otros trabajos de nuestro grupo (Castillo, Benítez y Padilla, 2009; CastilloDíaz, Padilla-García, Gómez-Benito y Andrés-Valle, 2010). También la utilización de otras metodologías (v.g. grupos focales con médicos, enfermeros, pacientes, etc.) podrían haber contribuido a enriquecer la definición del constructo. Sin embargo, la falta de tiempo y de recursos conllevaron que todas esas aportaciones no estuvieran a nuestro alcance.

Es cierto que lo que se presenta en este trabajo no incluye, por razones evidentes de espacio, los resultados de otros estudios que se han realizado con los ítems de la escala; análisis cuantitativo de ítems, evidencias de la estabilidad de las puntuaciones y consistencia interna (fiabilidad), obtención de evidencias de validez (relaciones con otros constructos establecidas en la definición sintáctica). Se comentará brevemente y de manera muy general, que los resultados obtenidos indican la idoneidad de los ítems desarrollados hasta el momento (consultar De los Santos-Roig, 2009), aunque el trabajo debe continuar con la aplicación del instrumento a otras enfermedades. Dicho esto, y como resultado de las fases objeto de análisis de este trabajo, se presenta en el Anexo, con la intención de que pueda ser consultada, la Primera Versión de la Escala ERCE.
Carretero-Dios, H. y Pérez, C. (2005). Normas para el desarrollo y revisión de estudios instrumentales. International Journal of Clinical and Health Psychology, 5, 521-551.

Carretero-Dios, H. y Pérez, C. (2007). Normas para el desarrollo y revisión de estudios instrumentales: consideraciones sobre la selección de tests en la investigación psicológica. International Journal of Clinical and Health Psychology, 7, 863-882.

Carretero-Dios, H., Pérez-Meléndez, C. y Buela-Casal, G. (2009). Content Validity and Metric Properties of a Pool of Items Developed to Assess Humor Appreciation. Spanish Journal of Psychology, 12, 773-787.

Castillo, M. Benítez, I. y Padilla, J. L. (2009, Septiembre). Obtención de evidencias de validez para un cuestionario sobre "apoyo familiar" mediante entrevistas cognitivas. Comunicación presentada en el XI Congreso de Metodología de las Ciencias Sociales y de la Salud. Málaga, España. 
Castillo-Díaz, M., Padilla-García, J. L., Gómez-Benito, J. y Andrés-Valle, A. (2010). A productivity map of cognitive pretest methods for improving survey questions. Psicothema, 22, 475-481.

De los Santos-Roig, M. (2009). Evaluación de la Representación de la Enfermedad: Creación de la Escala para la Representación Cognitiva de la Enfermedad, ERCE (Tesis doctoral).Universidad de Granada, Granada.

De los Santos-Roig, M., Pérez, C., Ruiz-González, I., Guardia-Archilla, T. y Martínez-García, A. (2010, Julio). Atribuciones Causales en la enfermedad: La importancia de las atribuciones especificas en la relación con otros componentes de la Representación de la Enfermedad, el Afrontamiento y el Ajuste del paciente. Póster presentado en el VII Congreso Iberoamericano de Psicología, Oviedo, España.

Diefenbach, M. A. y Leventhal, H. (1996). The common-sense model of illness representation: Theoretical and practical considerations. Journal of Social Distress and the Homeless, 5, 11-38.

French, D. y Weinman, J. (2008). Assessing illness perceptions: Beyond the IPQ. Psychology and Health, 23, 5-9.

Hagger, M. y Orbell, S. (2003). A meta-analytic review of the common-sense model of illness representations. Psychology and Health, 18, 141-184.

Haynes, S.N., Richard, D. C. S. y Kubany, E. S. (1995). Content validity in psychological assessment: a functional approach to concepts and methods. Psychological Assessment, 7, 238-247.

Heijmans, M. J. W. y de Ridder, D. (1998). Assessing illness representations of chronic illness: Explorations of their disease-specific nature. Journal of Behavioral Medicine, 21, 485-503

Lau, R. R. y Hartman, K. A. (1983). Common sense representations of common illnesses. Health Psychology, 2, 167-185.

Leventhal, H., Benyamini, Y., Brownlee, S., Diefenbach, M., Leventhal, E. A., Patrik-Miller, L. y Robitaille, C. (1997). Illness Representations: Theoretical Foundations. En K. J. Petrie y J. A. Weinman, (Eds.), Perceptions of Health and Illness (pp. 19-46). Amsterdam: Harwood Academic Publishers.

Leventhal, H., Brissette, I. y Leventhal, E. A. (2003). The Common-Sense Model of self-regulation of health and illness. En L. D. Cameron y H. Leventhal, (Eds.), The self-regulation of health and illness behaviour (pp. 4265). London y New York: Routledge.

Moss-Morris, R., Weinman, J., Petrie, K.J., Horne, R., Cameron, L. D. y Buick, D. (2002). The revised illness perception questionnaire (IPQ-R). Psychology and Health, 17, 1-16.

Muñiz, J. y Fonseca-Pedrero E. (2008). Construcción de instrumentos de medida para la evaluación universitaria. Revista de Investigación en Educación, 5, 13-25.

Nunnally, J. C., Bernstein, I. J. (1995). Análisis Factorial I: El modelo general y la condensación de la varianza. En Nunnally, J. C. y Bernstein, I. J., Teoría de la Psicometría. New York: MacGraw Hill.
Osterlind, S. J. (1989). Constructing test items. Boston: Kluwer Academic Publishers.

Pérez-Gil, J. A., Chacón-Moscoso, S. y Moreno-Rodríguez, R. (2000). Validez de constructo: el uso del análisis factorial exploratorioconfirmatorio para obtener evidencias de validez. Psicothema, 12, 442446.

Prieto, G. y Delgado, A. R. (1996). Construcción de los ítems. En J. Muñiz, (Ed.), Psicometría (pp. 139-170). Madrid: Universitas.

Quiceno, J. M. y Vinaccia, S. (2010). Percepción de enfermedad: una aproximación a partir del "Illness Perception Questionnaire". Psicología desde el Caribe, 25, 56-83.

Rubio, D. M., Berg-Weger, M., Tebb, S. S., Lee, E. S. y Rauch, S. (2003). Objectifying content validity: Conducting a content validity study in social work research. Social Work Research, 27, 94-104.

Scharloo, M. y Kaptein, A. (1997). Measurement of illness perceptions in patients with chronic somatic illness: a review of the literature. En K. J. Petrie y J. Weinman, (Eds.), Perceptions of health and illness: current research and applications (pp. 103-135). London: Harwood.

Shiloh, S., Rashuk-Rosenthal, D. y Benyamini, Y. (2002). Illness Causal Attributions. An exploratory study of their structure and associations with other illness cognitions and perceptions of control. Journal of Behavioral Medicine, 25, 373-394

Skinner, E. A. (1996). A guide to constructs of control. Journal of Personality and Social Psychology, 71, 549-570.

Smith, G. T., Fischer, S., y Fister, S. M. (2003). Incremental validity principles of test construction. Psychological Assessment, 15(4), 467-477.

Turk, D. C., Rudy, T. E. y Salovey, P. (1986). Implicit models of illness. Journal of Behavioral Medicine, 9, 453-474.

Van-der Hofstadt, C. J. y Rodríguez-Marín, J. (1997). Adaptación de un cuestionario para la medida de la representación de la enfermedad. Psicothema, 9, 237-245.

Willis, G. B. (2004). Cognitive Interview Revisiting: A useful technique, in Theory? En S. Presser, J. Rothgeb, M. P. Couper, J. T. Lessler, E. Martin, J. Martin y E. Singer (Eds.), Methods for Testing and Evaluating Survey Questions (pp. 23-43). Nueva Jersey: John Wiley and Sons.

Walker, J. (2001). Control and the Psychology of Health. Philadelphia: Open University Press.

Weinman, J., Petrie, K.J. Moss-Morris, R. y Horne, R. (1996). The illness perception questionnaire: a new method for assessing the cognitive presentations of illness. Psychology and Health, 11, 431-440.

(Artículo recibido: 10-11-2011, revisado: 25-05-2012, aceptado: 25-05-2012) 
Anexo. Versión de la ERCE tras el análisis de ítems

\section{ESCALA IDENTIDAD}

A continuación aparece una lista con una serie de síntomas. Queremos que nos indiques cuáles de ellos ESTÁN PRESENTES EN TU VIDA, es decir padeces FRECUENTEMENTE, AUNQUE NO SEA DIARIAMENTE. Para ello, marca con una cruz la casilla que aparece junto a cada uno. Posteriormente, sobre cada síntoma marcado, queremos que nos indiques si crees que está relacionado o no con TU DIABETES. Si tienes alguna duda, levanta la mano y te atenderé de inmediato.

\begin{tabular}{|c|c|c|c|}
\hline MARCA CON UNA CRUZ & \multicolumn{3}{|c|}{$\begin{array}{l}\text { ¿Crees que los síntomas que has marcado } \\
\text { stán relacionados con TU Diabetes? }\end{array}$} \\
\hline Sudoración & SI & NO & A VECES \\
\hline Temblores & SI & NO & A VECES \\
\hline Nerviosismo & SI & NO & A VECES \\
\hline Hambre & SI & NO & A VECES \\
\hline Debilidad & SI & NO & A VECES \\
\hline Palpitaciones & SI & NO & A VECES \\
\hline Ardores & SI & NO & A VECES \\
\hline Hormigueo (en la boca, en los hombros, etc.) & SI & NO & A VECES \\
\hline Nauseas & SI & NO & A VECES \\
\hline Dolor de cabeza & SI & NO & A VECES \\
\hline Dificultad para hablar o moverse & SI & NO & A VECES \\
\hline Agresividad & SI & NO & A VECES \\
\hline Problemas para ver bien & SI & NO & A VECES \\
\hline Desorientación & SI & NO & A VECES \\
\hline Mareo & SI & NO & A VECES \\
\hline Cansancio o sueño & SI & NO & A VECES \\
\hline Mucha sed, boca seca & SI & NO & A VECES \\
\hline Respiraciones rápidas y profundas & SI & NO & A VECES \\
\hline Piel seca & SI & NO & A VECES \\
\hline Otras & SI & NO & A VECES \\
\hline
\end{tabular}

ESCALA CAUSAL

Como sabes, las enfermedades pueden estar causadas por diversos factores o situaciones. Ahora queremos que nos indiques, de todas las causas que se enumeran a continuación, aquéllas que tú crees que han favorecido que tu enfermedad se desarrollara en un principio, es decir, que tú consideres que fueron las que participaron en el origen de tu enfermedad. Después, queremos que pienses en los síntomas y en cuáles de las causas que aparecen pueden, a tu juicio, desencadenar o estar presentes en la aparición de los síntomas que habitualmente padeces. Para ello, junto a cada causa aparece una casilla. Marca con una cruz la causa que creas está relacionada con el origen de la enfermedad o con la aparición de sintomatología. Por supuesto, si no crees que esa causa tenga nada que ver ni con la enfermedad ni con los síntomas, déjala sin tachar. Si tienes alguna duda, levanta la mano y te atenderé de inmediato.

\section{ENFERMEDAD}

Causas Medioambientales (polución, agentes tóxicos, etc.)

Causas Biológicas (Edad, Hereditaria, Virus, Enfermedades, etc.)

Estilos de Vida (Hábitos de vida, de comida, de sueño, rutina de trabajo, etc.)

Causas Psicológicas (Estrés, la manera de tomarse las cosas, las preocupaciones, etc.)

Atención Médica (Escasa prevención de enfermedades, no hacerte chequeos médicos, no seguir las recomendaciones, malas condiciones de salud, etc.)

Causas Sobrenaturales (Dios, el destino, el mal de ojo, etc.)

Otras................................. INICA CUÁL O CUÁLES

\section{SÍNTOMAS}

Haber tenido un mal día

Haber dormido mucho o poco

Haberse saltado el tratamiento

Haberse saltado la dieta (por no comer o comer demasiado o inadecuadamente)

El haber hecho mucho o poco ejercicio ese día

El estar pasando por malos momentos

El estar preocupado por alguna razón (familiar, laboral, etc.) 
El estar pasando por una situación estresante (en casa, en el trabajo, etc.)

El estar desanimado, triste, sentirse solo, sin fuerzas, etc.

Otras................................NDICA CUÁL O CUÁLES

\section{ESCALA CONSECUENCIAS}

Ahora, nos interesa saber cuáles son las consecuencias que la Diabetes tiene sobre ti y para ello vamos a centrarnos en este momento SOLO en un aspecto de ésta. Concretamente queremos que pienses en TUS SÍNTOMAS, y en qué consecuencias tienen éstos sobre ti o sobre tu vida Cuando hablamos de síntomas nos referimos a aquellos propios de la enfermedad que aparecen en tu día a día. Sabemos que hay momentos del día en los que te encuentras mal (temblores, mareo, sudoración, etc.) y, por ejemplo, te despiertas en medio de la noche, o momentos en los que debes dejar de hacer lo que estabas haciendo hasta recuperarte.

Sabemos que diariamente puedes padecer cualquiera de estos síntomas y queremos saber si esas sensaciones te repercuten de alguna manera en tu vida y en tu entorno. Sobretodo queremos saber en qué grado te afectan. Para ello se te van a presentar una serie de preguntas que deberás responder, rodeando con un círculo un número entre el 1 y el 5 , de manera que 5 significaría que los síntomas te afectan mucho y 1 que no te afectan nada

Siguiendo estas instrucciones, lee las siguientes preguntas y marca tu respuesta. Por favor, no dejes ninguna pregunta sin contestar.

- De 1 a 5, ¿cómo afectan los sintomas de tu diabetes a tu propia familia?

- ¿Y en tus relaciones sociales?

- Piensa en tu trabajo u obligaciones diarias sean éstas como sean, es decir, remuneradas o no, domésticas, laborales, etc. ¿Cómo te afectan los sintomas de la enfermedad?

- Indica ahora, ¿cómo te afectan personalmente los síntomas de esta enfermedad, es decir, a ti mismo como persona; en tu estado de ánimo, en tu manera de ver la vida, en tus proyectos, etc.?

ESCALA DE RESPUESTA: No afectan nada (1) - Afectan mucho (5)

Siguiendo con la misma lógica que en el caso anterior, nos gustaría ahora que pensaras sólo en cómo tu enfermedad, como tal, se presenta diariamente en tu vida desde que fue diagnosticada. Es decir, nos referimos a cómo la enfermedad “cambia” o se va comportando durante el día, por ejemplo, con la aparición de los síntomas en determinados momentos (por la noche, por la mañana, etc.).

Bien, todo ello, al igual que ocurría anteriormente con los síntomas, puede afectarte de alguna manera y en concreto, tanto a ti personalmente como a diversos aspectos de tu vida. Por eso, ahora vamos a preguntarte por el grado en el que ese comportamiento diario de la enfermedad te afecta a ti y a cuanto te rodea. Recuerda que para contestar deberás rodear un número del 1 al 5 , donde el 5 sería indicativo de que te afecte mucho y el 1 de que no te afecte nada.

- ¿En qué grado afecta la evolución diaria de tu enfermedad a tu familia?

- ¿Y en tus relaciones sociales?

- Piensa en su trabajo u obligaciones diarias sean éstas como sean, es decir, remuneradas o no, domésticas, laborales, etc. ¿Cómo te afecta la evolución diaria de la enfermedad

- Indica ahora, ¿cómo te afecta personalmente, es decir, a ti mismo como persona; en tu estado de ánimo, en tu manera de ver la vida, en tus proyectos, etc.? ESCALA DE RESPUESTA: No afecta nada (1) - Afecta mucho (5)

Ahora nos vamos a centrar en las estrategias que pones en marcha para manejar tu enfermedad Es decir, nos centraremos en todo aquello que haces para mantenerla controlada, como por ejemplo, los análisis continuos de sangre, la dieta restrictiva, pincharte la insulina antes de cada comida, comer a determinadas horas, rutinas de vida que no alteren tu ritmo cotidiano. Estas conductas o estrategias que debes realizar, pueden afectarte a ti o a algunos aspectos de tu vida en general.

Indica, como en las preguntas anteriores, el grado en el que te afectan todas estas situaciones anteriormente descritas. Para ello usa como siempre, los números del 1 al 5 que aparecen junto a cada pregunta.

- ¿En qué grado afecta a tu familia todo lo que tienes que hacer para controlar tu enfermedad?

- ¿Y en tus relaciones sociales?

- Piensa en tu trabajo, ¿en qué grado te afecta todo lo que necesitas hacer por tu enfermedad?

- Indica ahora ¿cómo te afecta todo esto personalmente, es decir, a ti mismo como persona; en tu estado de ánimo, en tu manera de ver la vida, en tus proyectos, etc?

ESCALA DE RESPUESTA: No afecta nada (1) - Afecta mucho (5)

Como sabes, hasta el día de hoy tu enfermedad puede ser considerada como crónica. Este hecho puede hacerte ser capaz de anticipar determinados efectos, así como diversas consecuencias que tu enfermedad pueda tener en ti o en tu vida en general (p. ej. Dejar de hacer cosas que ahora haces, pensar que tu vida va a ser peor de lo que pudiera ser ahora, etc.).

Como en los casos anteriores, nos interesa evaluar esos aspectos, y para ello te presentamos distintas preguntas. Di el grado en que crees que te afectará tu enfermedad A LARGO PLAZO, marcando un número del 1 al 5 . Recuerda que es necesario que 
contestes a todas las preguntas.

- ¿En qué grado crees que los efectos y consecuencias originadas por la enfermedad te afectarán personalmente, en un futuro?

- ¿En qué grado crees que tu enfermedad afectará a tu familia?

- ¿Y en tu trabajo?

- ¿Y en tus relaciones sociales en general?

ESCALA DE RESPUESTA: No afectará nada (1) - Afectará mucho (5)

\section{ESCALA CONTROL-CURA}

Antes te hemos preguntado por las consecuencias que tiene para ti tu Diabetes.También hemos hablado del control y de cómo esa forma de controlar la enfermedad (pincharse, comer a determinadas horas, etc.) puede afectar a tu vida o puede tener determinado impacto sobre ti y tu entorno.

Ahora vamos a preguntarte también por algo relacionado con el control, pero en este caso únicamente queremos que nos indiques lo controlable que te parece que es tu enfermedad, es decir, nos queremos referir a si crees que existen estrategias que permitan o que hagan posible mantenerla bajo control, estable, manejada, etc. No hace falta que pienses en quién debe poner en marcha estas estrategias, o si cuando las ha puesto en marcha, han funcionado. Sólo queremos que pienses en qué grado es posible controlar la enfermedad.

Para indicar todo esto se te van a presentar una serie de preguntas, a las que deberás responder rodeando con un círculo un número del 1 al 5 igual que en ocasiones anteriores (sabiendo que 5 significa que es muy controlable y 1 que no es nada controlable)

- De 1 a 5, jen qué grado crees que tu enfermedad es controlable?

- Y los síntomas con que se manifiesta tu enfermedad, ¿en qué grado crees que pueden controlarse ESCALA DE RESPUESTA: Nada controlable/s (1)-Muy controlable/s (5)

Ahora queremos que pienses en lo que TU puedes hacer para controlar la enfermedad Es decir nos interesa conocer tu opinión sobre el grado en que crees que tu enfermedad o diversos aspectos de ésta DEPENDEN de lo que TÚ PERSONALMENTE hagas, es decir, están bajo tu control (por ejemplo, hacer ejercicio, llevar una dieta saludable, hacerse análisis continuos, tomarse las cosas con calma, etc.)

Para ello, se te van a presentar una serie de preguntas que, como anteriormente, deberás responder rodeando con un círculo el número del 1 al 5 que represente mejor tu opinión. Recuerda no dejar ninguna pregunta sin contestar.

- De 1 a 5, zen qué grado crees que el control de tu enfermedad depende de lo que hagas?

- Y el manejo de los síntomas que aparecen diariamente, zen qué grado depende de ti?

- Como ya nos indicaste anteriormente, conoces en alguna medida las consecuencias que provoca o puede provocar tu enfermedad. $\dot{i}$ En qué grado crees que puedes controlarlas?

- Como sabes, los síntomas de tu enfermedad son provocados por diversos factores (p. ej. el estrés, alteraciones emocionales, alimentación, etc.). De 1 a 5, indica en qué grado puedes hacer algo para controlar algunos de esos factores

ESCALA DE RESPUESTA: No depende (1) - Depende Totalmente (5)

Como sabes, tu diabetes requiere un tratamiento médico y conductual concreto (hacer ejercicio, dieta estricta, etc ) que debes seguir y que es fundamental para mantener tu enfermedad bajo control. Sin embargo, sabemos que a veces los síntomas aparecen, o la enfermedad se descontrola, a pesar de que tú estés siguiendo las recomendaciones médicas. Como sabemos que eso puede ocurrir, queremos conocer tu opinión sobre el grado en que crees que tu enfermedad y diversos aspectos de ésta, está bajo control del TRATAMIENTO, es decir, nos interesa saber hasta qué punto crees que el tratamiento SIRVE para manejar o mantener controlada tu diabetes.

Como en los casos anteriores, se presentan una serie de preguntas que debes responder usando las números del 1 al 5 con el sentido explicado anteriormente Recuerda que es necesario no dejar ninguna pregunta sin contestar

- Piensa en cómo la enfermedad se manifiesta diariamente (altibajos, bajadas y subidas de "azúcar", etc.). De 1 a 5 , zen qué grado crees que el tratamiento sirve para controlar la aparición de estos sintomas?

- Como sabes, muchos de esos altibajos a los que nos referimos pueden estar motivados por causas como el estrés, la ansiedad, emociones como la tristeza, el enfado, etc. También decíamos antes que el tratamiento incluye aspectos médicos, pero también recomendaciones relativas a tu comportamiento (por ejemplo, debe tener unos hábitos de vida saludable, hacer ejercicio físico, llevar una dieta estricta, etc.). De 1 a 5 , ¿̇hasta qué punto crees que estas recomendaciones sirven para controlar esos factores que pueden dar lugar a los altibajos?

- Además de los síntomas diarios que seguramente padeces, sabes que tu enfermedad puede provocarte algunos trastornos físicos en el futuro. De 1 a 5 , ¿hasta qué punto crees que las recomendaciones relativas a tu comportamiento (ejercicio físico, dieta adecuada, etc) te podrían servir para paliar o reducir dichos trastornos en un futuro?

ESCALA DE RESPUESTA: No sirve en absoluto (1)-Sirve totalmente (5) 
Ahora nos interesa que pienses en las personas que de alguna manera te ayudan a sobrellevar tu enfermedad (médico, cuidador, familia, etc.). Sabemos que muchas veces son estas personas, las que te ayudan de alguna manera a controlar la enfermedad (por ejemplo, tu pareja puede cocinar la comida respetando la dieta, o puede animarte a hacerte un análisis, o tu médico puede mostrar interés en cómo estás cada vez que lo ves, prescribiéndote pautas nuevas, educándote en tu enfermedad, etc.). Como ves, estas personas pueden participar en los cuidados cotidianos que necesitas por tu enfermedad. Queremos que nos indiques, en qué medida crees que ESTAS PERSONAS pueden ayudarte a CONTROLAR tu diabetes.

Como en los casos anteriores, se presentarán una serie de preguntas que debes responder usando la escala numérica explicada anteriormente. Recuerda que es necesario no dejar ninguna pregunta sin contestar.

- De 1 a 5, ¿'hasta qué punto crees que otras personas te ayudan a controlar de alguna manera los sintomas diarios de tu enfermedad (p.e. animándote a hacerte un análisis, cocinar la comida respetando tu dieta, etc.?

- Como sabes, gran parte de ese malestar diario al que nos referimos puede haber sido provocado, o estar precedido de situaciones que te han alterado de alguna manera (el estrés, alguna discusión, estado de ánimo decaído, que hayas comido algo inadecuado, etc.). De 1 a 5 , zen qué grado crees que otras personas pueden ayudarte a controlar esas situaciones que pueden dar lugar a la aparición de síntomas?

ESCALA DE RESPUESTA: No ayudan en absoluto (1)-Ayudan totalmente (5)

Finalmente, queremos saber tu opinión acerca del grado en que crees que tu propia diabetes, no la diabetes en general, es curable, es decir, se puede solucionar. Utiliza para contestar los números del 1 al 5 que aparecen junto a la pregunta.

- De 1 a 5 , zen qué grado crees que tu enfermedad se puede curar?

ESCALA DE RESPUESTA: No se puede curar (1)- Se puede curar (5)

\section{ESCALA CURSO}

Nos interesa que nos digas lo que piensas sobre la duración de tu Diabetes, es decir, nos interesa conocer si crees que la diabetes va a durar poco, o si crees que estará presente durante mucho tiempo. Para que nos lo indiques, te vamos a presentar tres frases. Deberás decirnos cuál de las dos representa mejor tu opinión marcando con una cruz SOLO UNA DE ELLAS.

Mi enfermedad estará siempre presente $\square$ Mi enfermedad será pasajera $\square$ Mi enfermedad es imprevisible

También nos interesa saber tu opinión sobre cómo crees que va a evolucionar la enfermedad, así como los síntomas, conforme pase el tiempo, es decir, queremos que nos indiques si piensas que va a mantenerse estable, si crees que irá evolucionando a mejor, que irá empeorando, etc. Haz como en el caso anterior, marca SOLO UNA DE LAS OPCIONES

Mi enfermedad se mantendrá siempre como hasta abora

Mi enfermedad mejorará con el tiempo
Mi enfermedad empeorará progresivamente

Mi enfermedad irá cambiando por temporadas 